\title{
CLASS NUMBERS OF POSITIVE DEFINITE QUATERNARY FORMS ${ }^{1}$
}

\author{
BY PAUL PONOMAREV ${ }^{2}$ \\ Communicated by Nathan Jacobson, December 1, 1969
}

1. Introduction. Let $V$ be a quadratic vector space over the field of rational numbers $Q$. We assume that the associated quadratic form $q$ is positive definite with square discriminant. Let $M$ be a lattice in $V$ which is maximal integral with respect to $q$. We denote by $H$ the number of proper classes of maximal integral lattices. The purpose of this note is to announce a formula for $H$. This formula is derived by applying the Selberg Trace Formula in an appropriate manner. The method we employ is motivated by the successful use of the Selberg Trace Formula in the computation of ideal class numbers of quaternion algebras over $\boldsymbol{Q}$ (cf. [6]).

Since $q$ has square discriminant, we may assume that $V=\mathfrak{A}$, a quaternion (division) algebra over $\mathbb{Q}$, and $q=N$, the norm form of $\mathfrak{A}$. We may take $M$ to be $\mathcal{\theta}$, a fixed maximal order in $\mathfrak{A}$. If $x_{1}, x_{2}, x_{3}, x_{4}$ is a basis of $\mathcal{O}$ over the ring of integers $\boldsymbol{Z}$, then the discriminant of $\mathcal{\theta}$ with respect to the norm form $N$ is $=\left|\operatorname{Tr}\left(x_{i} x_{j}^{*}\right)\right|=D$, the discriminant of the quaternion algebra $\mathfrak{A}$. Here ${ }^{*}$ is the canonical involution of $\mathfrak{A}$. It is well known that $D=d^{2}$, where $d$ is a positive square-free integer. Let us write $d=p_{1} \cdots p_{e}$, where the $p_{i}, i=1, \cdots, e$ are distinct prime numbers. We recall that $\left\{p_{1}, \cdots, p_{\theta}\right\}$ is the set of finite primes $p$ such that $\mathfrak{H}_{p}=\mathfrak{H} \otimes_{\mathrm{Q}} \boldsymbol{Q}_{p}$ is a division algebra over $\boldsymbol{Q}_{p}$, the field of $p$-adic numbers. One calls $p_{1}, \cdots, p_{e}$ the nonsplit or ramified primes of $\mathfrak{A}$.

We do not apply the Selberg Trace Formula in the setting afforded by the orthogonal groups which appear in the usual definition of $H$. The reason is that the definitions of these groups involve a norm condition which makes integration unmanageable and which also complicates conjugacy considerations. To avoid these difficulties, we replace the usual definition of $H$ by one which is more suitable for our

AMS Subject Classifications. Primary 1016, 1069, 1570; Secondary 1646, 2265.

Key Words and Phrases. Positive definite quaternary form, quadratic form, maximal integral lattices, square discriminant, quaternion algebra, Selberg trace formula.

1 Partially supported by an N.S.F. Graduate Fellowship.

2 The results announced here are part of the author's proposed Ph.D. thesis, written under the guidance of Professor T. Tamagawa at Yale University. 
purposes. A lattice $L$ in $\mathfrak{A}$ is said to be a normal ideal of $\mathfrak{A}$ if its left order (hence its right order) is maximal. Following [1], we say that two normal ideals $L$ and $M$ of $\mathfrak{A}$ are equivalent if there exist $\alpha, \beta \in \mathfrak{A}$ such that $M=\alpha L \beta$. One easily verifies that the number of equivalency classes of normal ideals of $\mathfrak{A}$ is finite and equal to $H$. It is this definition of $H$ which we use in our computations.

2. Statement of results. We first introduce some more notation. It is well known that the ideal class number of $\mathfrak{A}$ depends only on $D$ (cf. [3] and [6]). Let us denote it by $h_{d}$. For any integer $n$, denote by $h(\sqrt{ }-n)$ the class number of the quadratic field $Q(\sqrt{ }-n)$. For any positive integer $n$, set $\lambda(n)=$ the number of distinct primes which divide $n$, and define $\delta(\sqrt{ }-n)$ to be 1 if $n \mid d$ and there exists $x \in \mathfrak{A}$ such that $x^{2}+n=0$. Otherwise, define $\delta(\sqrt{ }-n)=0$.

THEOREM 2.1. (a) If $2 \nmid D$, then:

$$
H=2^{-e}\left(h_{d}^{2}+A_{3}^{2}+\sum_{m>3} \delta(\sqrt{ }-m) A_{m}^{2}\right)
$$

where

$$
\begin{aligned}
A_{3}=2^{e-1} \delta(\sqrt{ }-3), & A_{m}=2^{e-\lambda(m)-\sigma(m)} h(\sqrt{ }-m), \quad m>3, \text { and } \\
\sigma(m)= & -1 \quad \text { if } m \equiv 3(\bmod 8), \\
= & 0 \text { if } m \equiv 7(\bmod 8), \\
= & 1 \text { if } m \equiv 1(\bmod 4) .
\end{aligned}
$$

(b) If $2 \mid D$, then:

$$
H=2^{-e}\left(h_{d}^{2}+A_{1,2}^{2}+A_{3}^{2}+\sum_{m>3} \delta(\sqrt{ }-m) A_{m}^{2}\right)
$$

where

$$
\begin{aligned}
A_{1,2} & =2^{a-2}(\delta(\sqrt{ }-1)+\delta(\sqrt{ }-2)), \quad A_{3}=2^{\natural-2} \delta(\sqrt{ }-3), \\
A_{m} & =2^{a-\lambda(m)-\sigma(m)} h(\sqrt{ }-m) \quad \text { for } m>3, \quad \text { but now } \\
\sigma(m) & =1 \quad \text { if } m \equiv 2,3(\bmod 4), \\
& =2 \quad \text { if } m \equiv 1(\bmod 4) .
\end{aligned}
$$

REMARKs. (1) We recall that there is an explicit formula for $h_{d}$ in terms of the Legendre Symbol, namely:

$$
h_{d}=\frac{1}{12} \prod_{i=1}^{e}\left(p_{i}-1\right)+\frac{1}{4} \prod_{i=1}^{e}\left(1-\left(\frac{-1}{p_{i}}\right)\right)+\frac{1}{3} \prod_{i=1}^{e}\left(1-\left(\frac{-3}{p_{i}}\right)\right)
$$


where we make the convention:

$$
\left(\frac{-1}{2}\right)=\left(\frac{-3}{3}\right)=0, \quad\left(\frac{-3}{2}\right)=-1 \text {. }
$$

(2) If $2 \nmid D$ and $m \mid d$, the condition $\delta(\sqrt{ }-m)=1$ is equivalent to:

$$
\left(\frac{-m}{P_{j}}\right)=-1
$$

for all $p_{j}$ such that $\left(m, p_{j}\right)=1$. Using this, one can rephrase (a) of the theorem in terms of the Legendre Symbol, just as for $h_{d}$.

Corollary 2.2. Suppose $D=p^{2}, p$ a prime $>3$.

Then

where

$$
H=\frac{1}{2}\left(h_{p}^{2}+4^{f(p)} h(\sqrt{ }-p)^{2}\right),
$$

$$
\begin{aligned}
f(p) & =-1 \quad \text { if } p \equiv 1(\bmod 4), \\
& =0 \text { if } p \equiv 7(\bmod 8), \\
& =1 \text { if } p \equiv 3(\bmod 8) .
\end{aligned}
$$

We note that this formula closely resembles the formula for $t(p)$, the type number of $\mathfrak{A}$ (cf. [2]). It should also be remembered that the formula for $h_{p}$ is very simple: $h_{p}=(p-1) / 12$ if $p \equiv 1(\bmod 12)$; $(p+7) / 12$ if $p \equiv 5(\bmod 12) ;(p+5) / 12$ if $p \equiv 7(\bmod 12)$, and $(p+13) / 12$ if $p \equiv 11(\bmod 12)$.

3. Outline of the proof. For any associative ring $R$ with an identity, let $R^{*}=$ the group of invertible elements in $R$. For any group $S$, let $\Delta(S)$ be the diagonal subgroup of $S \times S$. Set $G=\left(\mathfrak{P}^{*} \times \mathfrak{H}^{*}\right) / \Delta\left(Q^{*}\right)$. If $s \in G$ and $\alpha, \beta \in \mathfrak{A}^{*}, s \equiv(\alpha, \beta)$ will mean $s \equiv(\alpha, \beta) \bmod \Delta\left(Q^{*}\right)$. Then $G$ acts on the set of normal ideals by: $s \equiv(\alpha, \beta): L \mapsto \alpha L \beta^{-1}$. For any normal ideal $L$, let $G_{L}=$ the isotropy group of $L$ under this action. Let $J_{\mathfrak{A}}$ be the idele group of $\mathfrak{A}, J_{\mathbf{Q}}$ the idele group of $\boldsymbol{Q}$, and define $J_{\mathscr{A}}^{1}$ $=\left\{\left.\left(\alpha_{p}\right) \in J_{\mathfrak{Q}}\left|\prod_{p}\right| N\left(\alpha_{p}\right)\right|_{p}=1\right\}, J_{\mathbf{Q}}^{1}=\left\{\left.\left(r_{p}\right) \in J_{\mathbf{Q}}\left|\prod_{p}\right| r_{p}\right|_{p}=1\right\}$. We then set $G_{\mathbf{A}}=\left(J_{\mathscr{P}}^{1} \times J_{\mathscr{Q}}^{1}\right) / \Delta\left(J_{\mathbf{Q}}^{1}\right) . G_{\mathbf{A}}$ acts on the set of normal ideals in the obvious manner: we identify each normal ideal $L$ with its corresponding $\tilde{L}=\prod_{p} L_{p}$, where $L_{p}=L \otimes_{z} \boldsymbol{Z}_{p}$, and $\boldsymbol{Z}_{p}=$ the ring of $p$-adic integers. Then if $\tilde{s} \in G_{\mathrm{A}}, \tilde{s} \equiv\left(\left(\alpha_{p}\right),\left(\beta_{p}\right)\right)$ takes $\tilde{L}$ to $\prod_{p} \alpha_{p} L_{p} \beta_{p}^{-1}$ $=\tilde{M}$ for some normal ideal $M$. Clearly, this action is transitive. Let $G_{\tilde{\mathcal{O}}}=$ the isotropy group of $\tilde{\mathcal{O}}$ under $G_{\mathrm{A}}$. We then have a double coset decomposition $G_{\mathrm{A}}=\bigcup_{k=1}^{H} G_{\tilde{\mathcal{O}}} \tilde{s}_{k} G=\bigcup_{k=1}^{H} G \tilde{s}_{\tilde{k}}^{-1} G_{\tilde{\mathcal{O}}}$, where the double coset $G \tilde{s}_{\mathfrak{k}}^{-1} G_{\tilde{\Theta}}$ corresponds to the class of the normal ideal $\tilde{s}_{\mathfrak{k}}^{-1} \tilde{\mathcal{O}}=\tilde{M}_{k}$. One easily verifies that $G_{\mathrm{A}}$ is a locally compact unimodular group, $G_{\tilde{\vartheta}}$ is 
an open compact subgroup, and $G$ is a discrete subgroup such that $G_{\mathbf{A}} / G$ is compact. We normalize the measure $\mu$ on $G_{\mathbf{A}}$ so that $\mu\left(G_{\tilde{O}}\right)$ $=1$. Using the notation of $[5]$, we see that $L_{2}\left(G_{\tilde{\mathcal{O}}} \backslash G_{\mathrm{A}} / G\right)$ is a vector space of dimension $H$ over the field of complex numbers $C$, and for every $F \in L\left(G_{\mathrm{A}}, G_{\tilde{o}}\right)$, the convolution operator: $f \mapsto F * f$, for $f \in L_{2}\left(G_{\tilde{\mathcal{O}}} \backslash G_{\mathrm{A}} / G\right)$, defines a linear endomorphism of $L_{2}\left(G_{\tilde{\mathcal{O}}} \backslash G_{\mathrm{A}} / G\right)$. The Selberg Trace Formula then gives us an expression for $\operatorname{Tr}(F)$. For any $s \in G$, define $\{s\}=$ the conjugacy class of $s$ in $G, G(s)=$ the centralizer of $s$ in $G$, and $G_{\mathbf{A}}(s)=$ the centralizer of $s$ in $G_{\mathbf{A}}$. Taking $F_{\tilde{\mathcal{O}}}=X_{G \tilde{\mathcal{O}}}=$ the characteristic function of $G_{\tilde{\mathcal{O}}}$ and applying the trace formula, we obtain:

$$
H=\operatorname{Tr}\left(F_{\tilde{\vartheta}}\right)=\sum_{\{s\}} v\left(G_{A}(s) / G(s)\right) \int_{G_{A} / G_{A}(s)} \psi_{s}(\bar{\gamma}) d_{s} \bar{\gamma},
$$

where $\psi_{s}(\bar{\gamma})=F_{\tilde{\mathcal{O}}}\left(\gamma s \gamma^{-1}\right)$ for $\gamma \in G_{\mathrm{A}}$.

In this sum we need only consider the conjugacy classes of elements $s$ lying in some $G_{M_{k}}, k=1, \cdots, H$. Hence there are essentially only four possibilities for $s$ :

(i) $s \equiv( \pm 1,1)$.

(ii) $s \equiv(1, u)$ or $(u, 1)$, where $u^{2}+1=0$ or $u^{2} \pm u+1=0$.

(iii) $s \equiv\left(u, u^{\prime}\right)$, where $u, u^{\prime}$ are as in (ii).

(iv) $s \equiv\left(z, z^{\prime}\right)$, where $z$ and $z^{\prime}$ are integral, $N(z)=N\left(z^{\prime}\right) \neq 1$ and $N(z) \mid d$.

The contributions to the trace of the conjugacy classes represented by cases (i), (ii) and (iii) give the term $2^{-e} h_{d}^{2}$ in our formula. The remaining terms are contributed by the conjugacy classes represented by case (iv).

\section{REFERENCES}

1. M. Deuring, Algebren, Ergebnisse der Mathematik und ihrer Grenzgebiete, Band 4, Springer-Verlag, Berlin and New York, 1935, pp. 89-90.

2. - Die Anzahl der Typen von Maximalordnungen einer definiten Quaternionenalgebra mit primer Grundzahl, Jber. Deutsch. Math. Verein. 54 (1950), 24-41. MR 12, 159.

3. M. Eichler, Über die Idealklassenzahl total definiter Quaternionenalgebren, Math. Z. 43 (1937), 102-109.

4. - Zur Zahlentheorie der Quaternionen-Algebren, J. Reine Angew. Math. 195 (1955), 127-151. MR 18, 297.

5. T. Tamagawa, On Selberg's trace formula, J. Fac. Sci. Univ. Tokyo Sect. I 8(1960), 363-386. MR 23 \#A958.

6. - Harmonic analysis on Adele groups, Mimeographed Notes taken by L. Goldstein, Advanced Science Seminar in Algebraic Groups, Bowdoin College, Me., 1968.

Yale University, New Haven, Connecticut 06520 